\title{
Cinematic rendering for virtual anatomy and pathoanatomy in cranio-maxillofacial trauma care
}

\author{
Raphael Stehrer ${ }^{1 *}$, Michael Malek ${ }^{1}$ and Franz A Fellner ${ }^{2,3}$ \\ ${ }^{1}$ Department of Cranio-Maxillofacial Surgery, Kepler University Hospital, Medical Faculty of the Kepler University Linz, Krankenhausstraße 9, 4020 Linz, Austria \\ ${ }^{2}$ Central Radiology Institute, Kepler University Hospital, Medical Faculty of the Johannes Kepler University, Linz, Austria \\ ${ }^{3}$ Medical Faculty of the Friedrich-Alexander-University of Erlangen-Nürnberg, Erlangen, Germany
}

\begin{abstract}
Background and objectives: Cinematic Rendering is a recently introduced post-processing method to generate three-dimensional reconstructions from twodimensional data sets produced by procedures such as computed tomography (CT) and magnetic resonance (MR) imaging. This paper describes initial clinical experiences with this new visualization method and discusses the potential advantages of Cinematic Rendering in comparison to Volume Rendering in the treatment of cranio-maxillofacial bone trauma.
\end{abstract}

Material and methods: The three patients described in this study suffered cranio-maxillofacial bone injuries due to trauma and were treated in our Department of Cranio-Maxillofacial Surgery. Over the course of their treatment, a CT scan of the skull was performed on each patient and a three-dimensional depiction was generated by means of Volume Rendering. For the purpose of this study, the CT imaging data were post-processed by means of Cinematic Rendering and threedimensional images were generated using this technique.

Conclusion: Cinematic Rendering significantly increases the quality of the three-dimensional post-processing; in contrast to standard Volume Rendering, it is possible to produce nearly photorealistic, detailed three-dimensional representations of the human body. Thus, especially for cranio-maxillofacial surgeons treating traumatic injuries, Cinematic Rendering has the potential to make it easier to diagnose fractures and abnormalities and to plan the corresponding operative procedures.

\section{Introduction}

In the field of cranio-maxillofacial surgery, radiological imaging, as an adjunct to clinical examination, is the decisive tool in the diagnosis and evaluation of cranio-maxillofacial trauma. If anamnesis or clinical examination suggests a severe injury, then standard medical practice calls for performing a computed tomography (CT) scan of the facial region to depict the extent of possible bone fractures or severe softtissue injuries. The cross-sectional views generated thereby-in axial orientation only, due to technical constraints-are then post-processed in order to compute the images used in everyday clinical practice. The images in sagittal and coronal orientation are generated from the axial layers using the multiplanar reconstruction (MPR) technique.

In addition to MPR, there are several other post-processing options, a few of which provide the observer with the possibility of generating three-dimensional images from the initially acquired two-dimensional data sets. Especially in the field of cranio-maxillofacial surgery, these three-dimensional images are regularly used to visualize complex craniofacial injuries and to plan surgical interventions. In going about this, the current standard three-dimensional visualization technique is volume rendering.

\section{Volume rendering}

A group of researchers at the Mayo Clinic in the USA in the 1970s made the first attempts to generate three-dimensional images from CT cross-sectional images. However, it took considerable technical progress with respect to computer hardware until, finally, Pixar in cooperation with a CT research group at Johns Hopkins Hospital in Maryland developed Volume Rendering, which then became an established post-processing method for three-dimensional depiction [1-4].

\section{Cinematic rendering}

Technical advances in the field of computer animation as well as new and improved algorithms recently led to the development of a novel method of three-dimensional visualization of radiological imaging data sets that enormously increases the quality of the threedimensional post-processing. This is based on software technology capable of generating practically photorealistic, three-dimensional images of the human body [5]. Since the developers were inspired by a technique used in animated filmmaking and the entertainment industry, they named the software Cinematic Rendering.

${ }^{\star}$ Correspondence to: Raphael Stehrer, Department of Cranio-Maxillofacial Surgery, Kepler University Hospital, Medical Faculty of the Johannes Kepler University, Linz, Austria, E-mail: Raphael.stehrer@kepleruniklinikum.at

Key words: virtual anatomy, virtual pathoanatomy, cinematic rendering, volume rendering, three-dimensional (3D) post-processing techniques, trauma care, computed tomography $(C T)$

Received: April 05, 2020; Accepted: April 13, 2020; Published: April 16, 2020 


\section{Objective of the study}

This paper describes initial clinical experiences with this new visualization method and discusses the potential advantages of Cinematic Rendering in comparison to Volume Rendering in the treatment of cranio-maxillofacial bone trauma in the field of craniomaxillofacial surgery.

\section{Material and methods}

\section{Study design}

The patients described in this study suffered cranio-maxillofacial bone injuries due to trauma and were treated in our Department of Cranio-Maxillofacial Surgery. Over the course of their treatment, all these patients underwent clinical examination that resulted in the presumption of an injury to a bony portion of the skull. A CT scan (layer thickness: $1.5 \mathrm{~mm}$ ) of the skull was performed on each patient and, as is standard operating procedure, a three-dimensional depiction was generated on the routine image post-processing unit (syngo.via) by means of Volume Rendering.

Afterwards, for the purpose of this study, the imaging data gathered in conjunction with these courses of treatment were post-processed by means of Cinematic Rendering and three-dimensional images were generated using this technique. This was done with a Cinematic Rendering version made available within the framework of a research platform (Syngo.Via Frontier, version.1.0.0, Siemens Healthineers). The gathered data as well as the post-processing via Cinematic Rendering were anonymized; authorization had been obtained from the local ethics commission of the State of Upper Austria (K-134-17).

\section{Volume rendering - technical background}

Generating three-dimensional images from cross-sectional data sets by means of Volume Rendering is a three-step process: volume formation, classification, and image projection. Volume formation entails gathering the image data and doing the general preparatory work on them-e.g. adjusting the size of each volume element (voxel) [6]. In the classification process, it is determined which type of tissue (bone, soft tissue, fat, etc.) will be depicted by each voxel, and each voxel is assigned a predefined opacity \& color value in order to specify a particular visual property. Volume rendering employs a percentagebased classification method (continuous) instead of a thresholdvalue-based method (binary). In order to specify which tissue will be represented to what percent by a voxel, for each voxel, a so-called trapezoid is used for each individual type of tissue [1,6-8].

The projection method used is ray casting, which simulates the projection of individual rays of light into the data set. The final image emerges from the interaction of the rays of light with the voxels, and, as a result of this interaction, depends on the color and transparency assigned to the respective voxels $[6,7,9]$.

\section{Cinematic rendering - technical background}

In contrast to the approach taken in Volume Rendering brightness, color \& opacity values predefined on the basis of a synthetic light source - and in contrast to the ray casting procedure, Cinematic Rendering simulates the propagation of natural light with the help of a physical, real-time rendering method. This algorithm uses a Monte Carlo path tracking method, and simulates the complex interplay of visible photons with the scanned anatomical depiction of the patient. Whereas the ray casting method used in Volume Rendering considers only emission and absorption of radiant energy along a single ray with simple diffusion, the Monte Carlo path tracking method is capable of generating photorealistic and even hyper-realistic images. To render them, a stochastic process is used to simulate light transport per pixel along thousands of photon paths through the anatomical depiction of the patient $[5,10,11]$.

Achieving this entails recording a so-called spherical panorama that makes it possible to register a scene's actual lighting conditions and to apply them to all elements that are inserted into this scene. Thanks to these high dynamic range (HDR) rendering lightmaps, computer-generated objects placed in these lightmaps can be accurately illuminated [12].

In comparison to the artificial light sources used in Volume Rendering, the combination of multiple and, thus, more precise simulation of the photons' dispersion and the interaction of the photons with the interiors of depicted structures allows for a more realistic, more natural lighting of the rendered data [10]. Moreover, the use of motion blur effects makes it possible to achieve fluid animation during camera movements and, with the help of depth-of-field effects, to highlight those anatomical structures that are of particular interest.

\section{Potential clinical applications}

In order to elaborate on the potential uses of Cinematic Rendering in the field of cranio-maxillofacial surgery for the evaluation and diagnosis of fractures in the craniofacial region, we will describe our initial clinical experiences on the basis of three patients who were treated in our unit.

\section{Patient 1}

A 65-year-old man was kicked in the face by a horse, and delivered by emergency rescue helicopter to our trauma surgery emergency room. The patient received treatment in accordance with typical interdisciplinary shock-room management, and a full body CT scan was performed according to international shock-room protocol. Radiological images and clinical examination yielded the diagnosis of a centro-lateral mid-face fracture which was treated operatively. Figures 1 and 2 show three-dimensional reconstructions that were generated by Volume Rendering; Figures 3 and 4 show three-dimensional reconstructions generated by Cinematic Rendering.

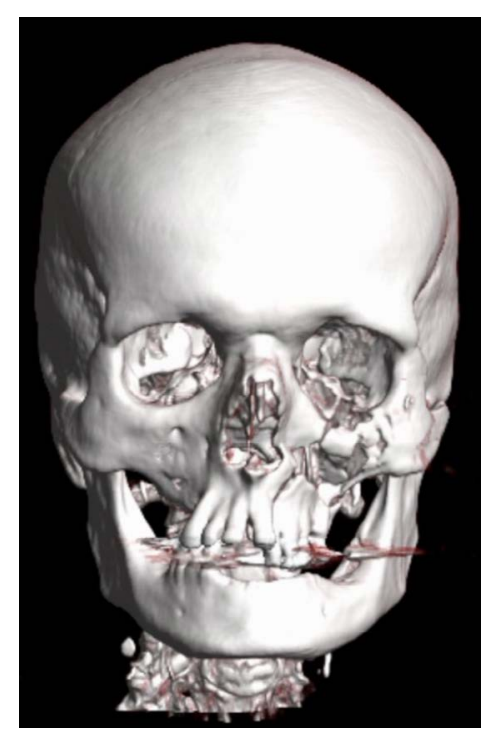

Figure 1. Three-dimensional reconstruction produced with Volume Rendering of a 65-year-old patient who suffered a centro-lateral mid-face fracture 


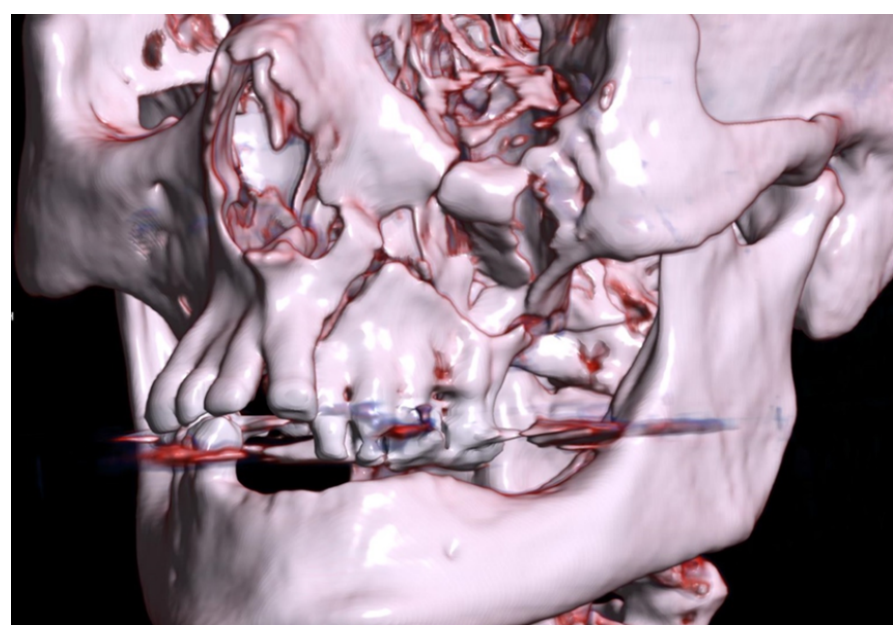

Figure 2. More detailed Volume Rendering view on the mid-face fracture of the same patient as in Figure 1

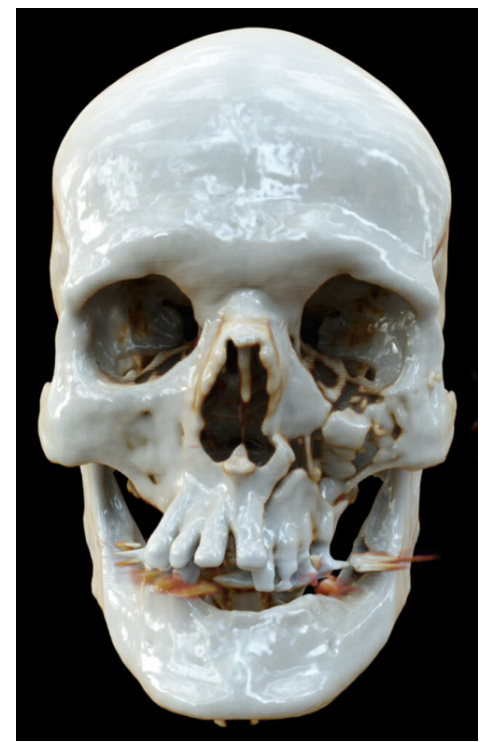

Figure 3. Three-dimensional reconstruction generated with Cinematic Rendering of data from the same patient who suffered a centro-lateral mid-face fracture as in Figures 1 and 2

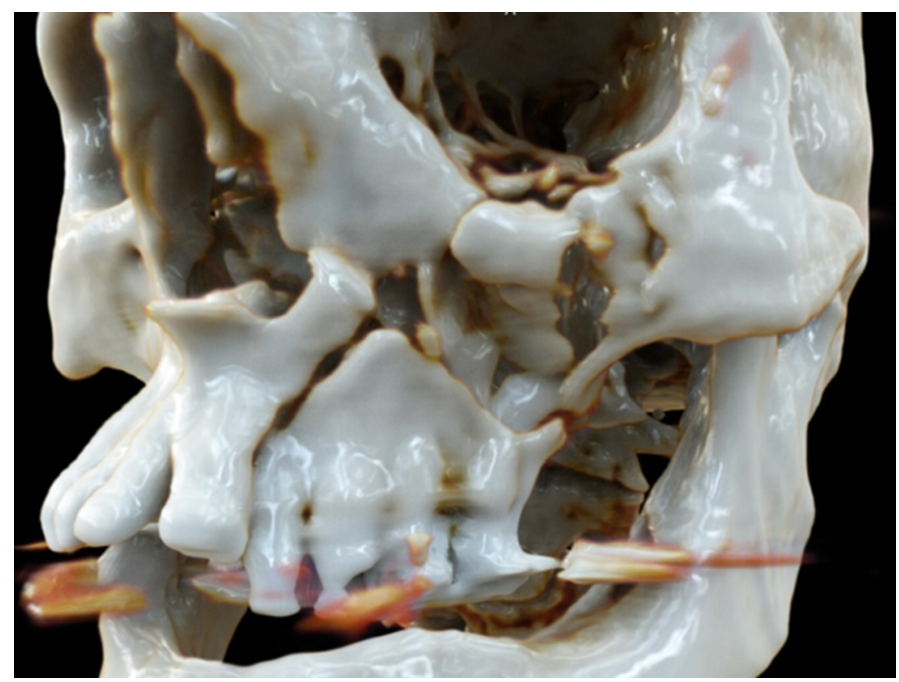

Figure 4. More detailed Cinematic Rendering view on the mid-face fracture of the same patient as in Figure 3

\section{Patient 2}

A 44-year-old man who had been in a bar fight walked into our emergency room. The ensuing clinical examination yielded suspicion that the patient had suffered a severe bone injury in the mandible region, so a CT scan of the entire skull was performed. In addition to the cross-sectional images in the three standard orientations (axial, sagittal and coronal), a three-dimensional reconstruction was done by means of Volume Rendering. The radiologist and the craniomaxillofacial surgeon who were on duty at the time diagnosed a fracture of the mandible in the area of the right angle of the jaw; this was also clearly visible in the three-dimensional reconstruction done by means of Volume Rendering (Figure 5).

The clinical examination also yielded suspicion of another fracture of the mandible in the area of Tooth 33, but there was no indication of a fracture in this area on either the two-dimensional cross-sectional images or the three-dimensional reconstruction (Figure 6).

Nevertheless, due to the clinical increased mobility as well as visible gingival bleeding, this region was explored during the course of the surgery, and this did indeed reveal a continuous, mobile fracture in addition to the fracture in the area of the right angle of the jaw. Therefore, both fractures were treated operatively by means of plate osteosynthesis.

In the three-dimensional reconstructions prepared in conjunction with this study using Cinematic Rendering, however, not only the fracture in the area of the right angle of the jaw (Figure 7) but also the fracture of the mandible in the area of Tooth 33 (Figure 8) can be seen clearly.

\section{Patient 3}

A 13-year-old boy suffered a syncope, whereby the patient collapsed and hit his chin on the ground. In our emergency room, the patient complained of severe pressure pain in the area of both mandibular joints, and he was already aware of extravasation from his right ear. A CT scan of the skull was performed, which, in addition to showing that no inter-cranial hemorrhaging had occurred, revealed the fracture of both mandibular joint heads with fragments tilted inwards. Due to the patient's young age, the fact that he was not prevented from opening his mouth, and the very cranial location of the fractures without a disturbance of the occlusion, a conservative treatment was preferred to surgery. Figures 9 and 10 show the three-dimensional reconstructions generated using Volume Rendering; Figures 11-14 show the threedimensional reconstructions generated using Cinematic Rendering.

\section{Discussion}

Cinematic Rendering makes it possible to generate photorealistic, detailed three-dimensional reconstructions of the human body on the basis of two-dimensional radiological cross-sectional data sets. In comparison to three-dimensional representations produced by means of Volume Rendering that appear rather glossy and plastic-like, images generated via Cinematic Rendering come across as more realistic, sharper and more lifelike. What is already empirically striking to observers viewing these images was also evaluated scientifically by Ebert et al. [9]. Questionnaires were filled out by 70 volunteers requested to assess three-dimensional reconstructions generated by Volume Rendering and Cinematic Rendering with respect to their degree of realism and other parameters. Here, Cinematic Rendering was judged to a statistically significant extent to be the more realistic procedure. The authors attribute this to a more complex illumination model used in Cinematic Rendering, and we concur with this conclusion. 


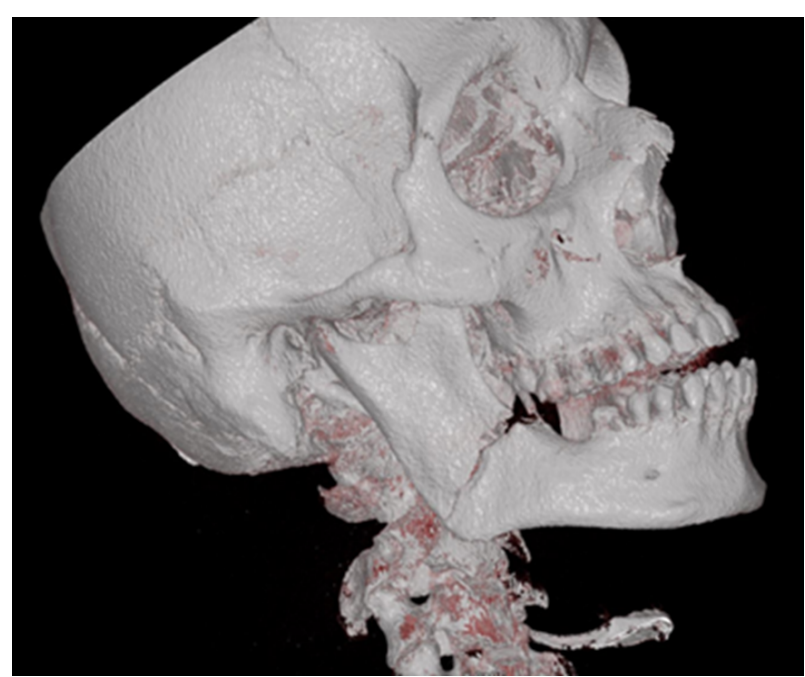

Figure 5. Three-dimensional reconstruction via Volume Rendering of the data of a 44-year-old patient who suffered fractures of the mandible both in the area of the right angle of the jaw, as well as in the area of Tooth 33. This Figure shows the fracture of the right angle of the jaw

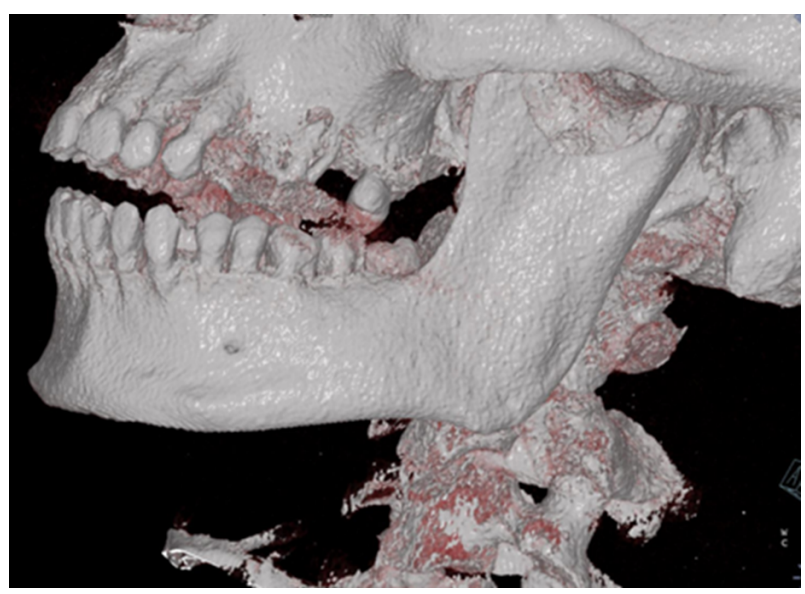

Figure 6. Three-dimensional Volume Rendering reconstruction of the patient in Figure 5 who suffered fracture of the mandible both in the area of the right angle of the jaw, as well as in the area of Tooth 33. However, here the bony tissue in the area of Tooth 33 is continuously intact so there is no indication of a fracture in this area

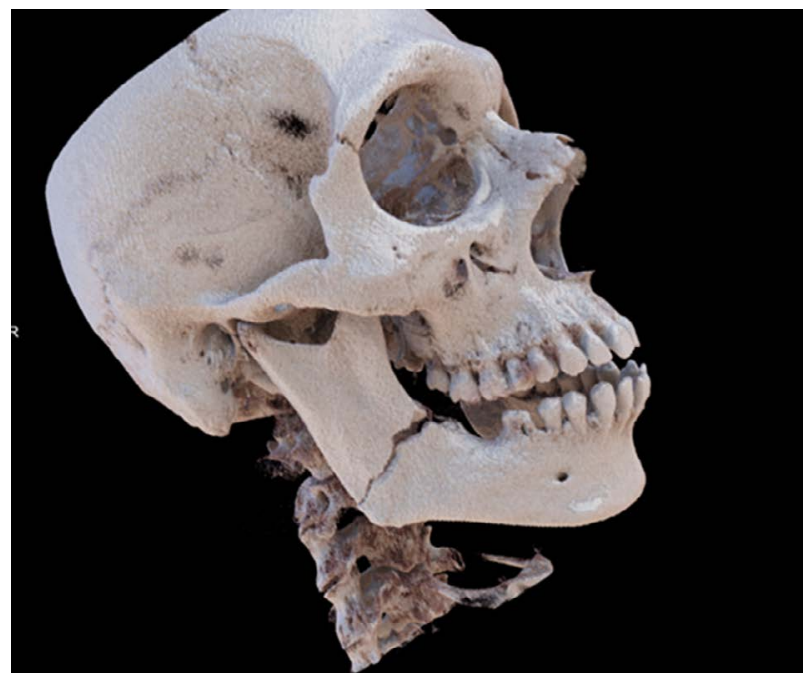

Figure 7. Three-dimensional reconstruction via Cinematic Rendering of the same patient as in Figures 5 and 6 demonstrating the fracture of the right angle of the jaw

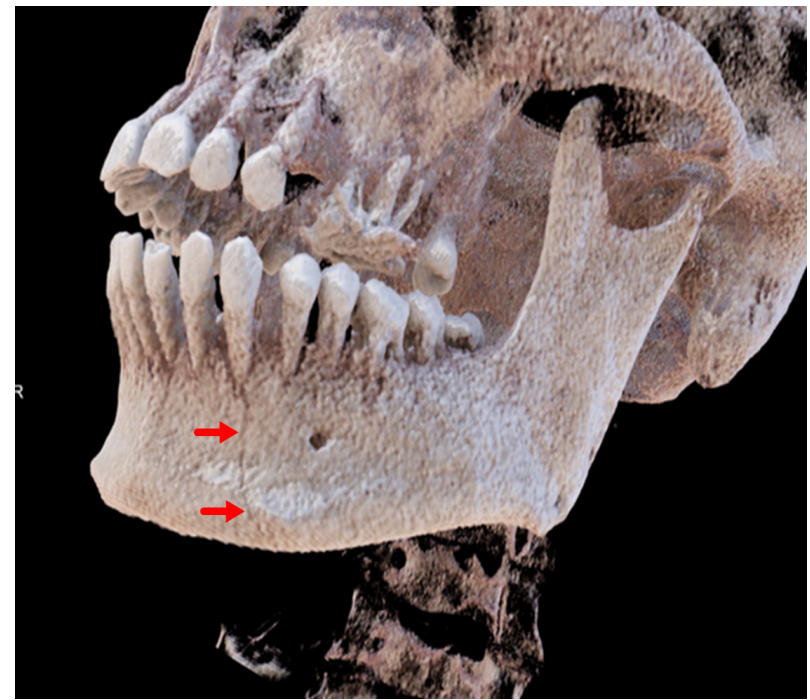

Figure 8. Three-dimensional reconstruction via Cinematic Rendering of the same patien as in Figure 7. Here, a fracture cleft in the area of Tooth 33 (arrow) is clearly demonstrated that could not be visualized by means of Volume Rendering

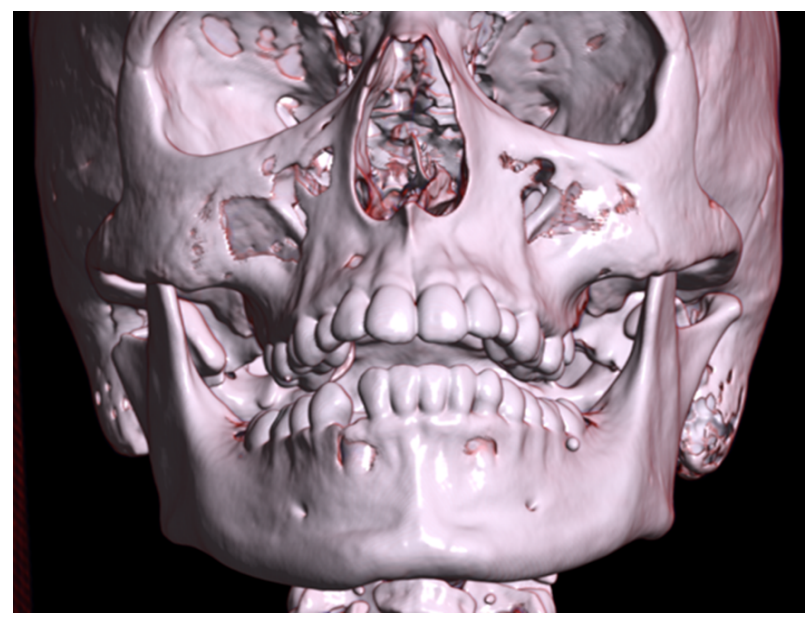

Figure 9. Three-dimensional reconstruction via Volume Rendering of the CT data of a 13-year-old patient who suffered a fracture of the mandibular condyles on both sides with inward-tilted fragments. Anterior view of the skull

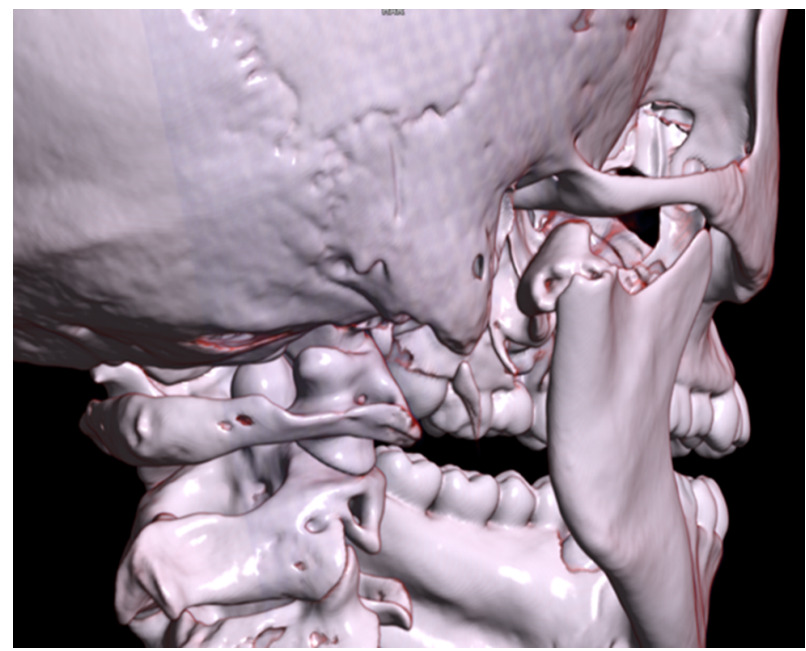

Figure 10. Three-dimensional reconstruction via Volume Rendering of the same patient as in Figure 9. This Figure gives a more detailed view from a posterior-lateral position on the fracture of the right mandibular condyle 


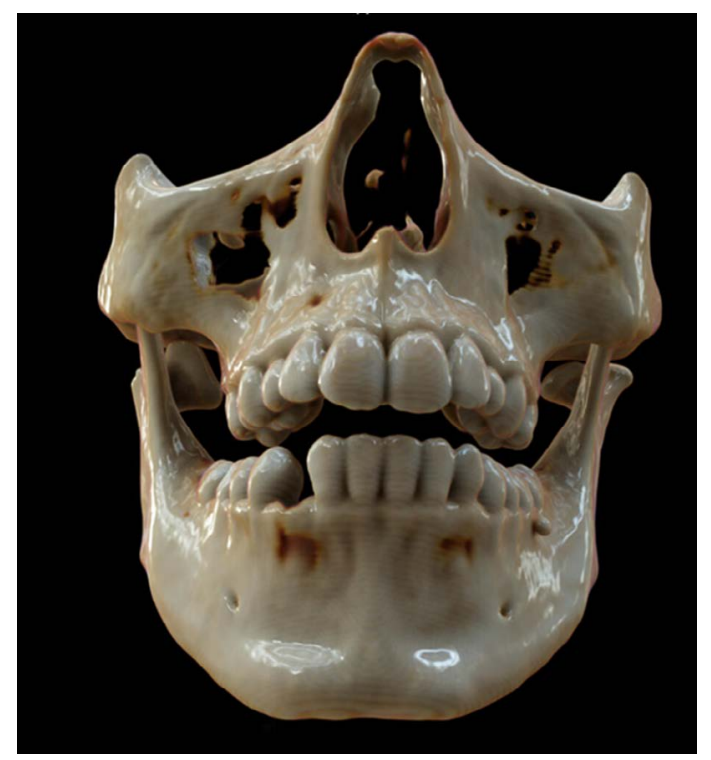

Figure 11. Three-dimensional reconstruction via Cinematic Rendering of the same patient as in Figures 9 and 10. Anterior view of the skull

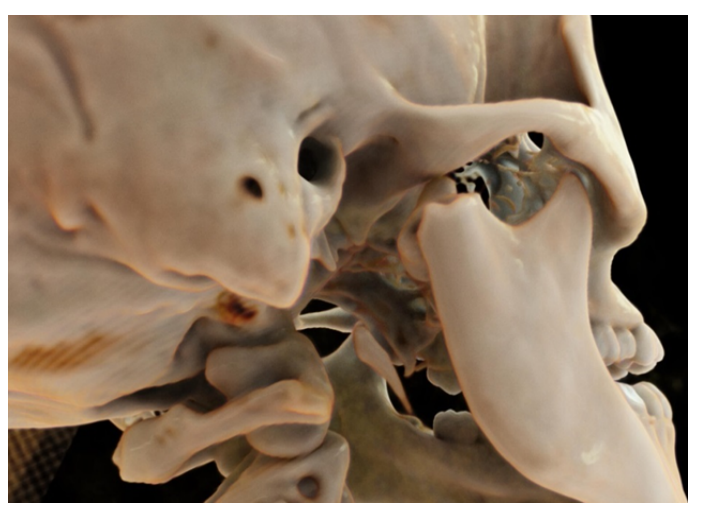

Figure 12. Three-dimensional reconstruction via Cinematic Rendering of the same patient as in Figure 11. In this detailed view from a posterior-lateral position on the fracture of the right mandibular condyle using Cinematic Rendering it is easier to evaluate the bony edges of the fractured fragments as well as the extent of the inward tilt

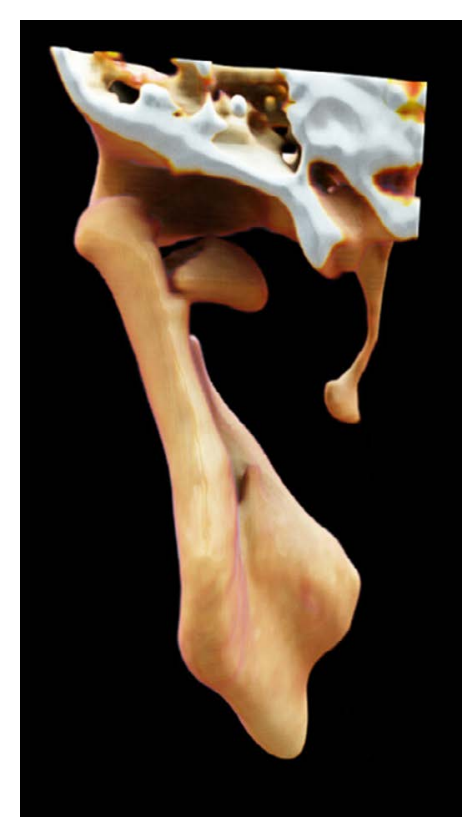

Figure 13. Three-dimensional reconstruction via Cinematic Rendering of the same patient as in Figure 11. This image provides a precise dorsal view of the inward-tilted fracture fragment of the left mandibular condyle 


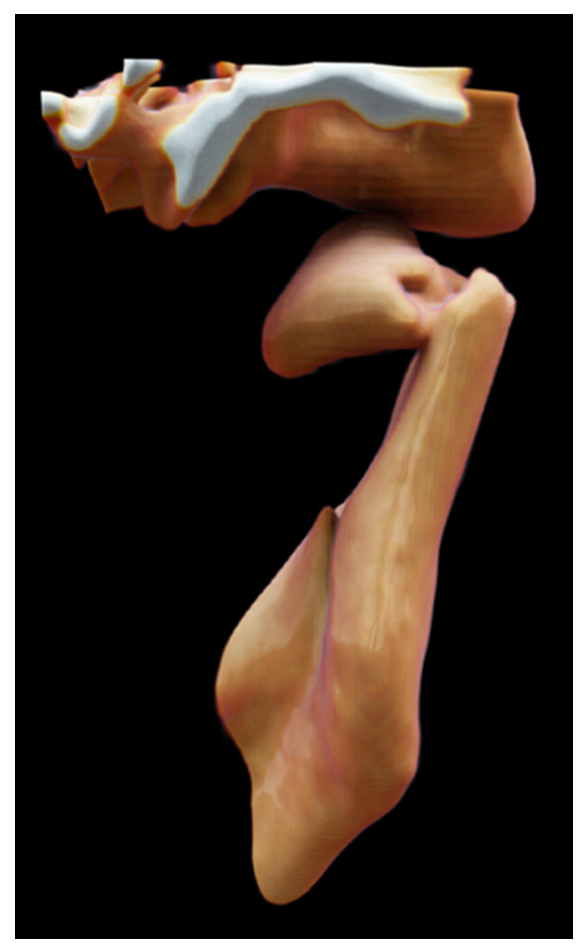

Figure 14. Three-dimensional reconstruction via Cinematic Rendering of the same patien as in Figure 11. This image provides a precise dorsal view of the inward-tilted fracture fragment of the right mandibular condyle

Due to its more complex illumination model and algorithm, Cinematic Rendering far surpasses all previous 3D methods in achieving more lifelike illumination. This results in complex transitions between well-lit and shadowy zones in three-dimensional representations, which enormously facilitates the human brain's process of registering possible anomalies. This is due to the fact that human beings perceive form \& depth information on the basis of light's interaction with an object $[13,14]$.

Naturally, three-dimensional representations computed from twodimensional data sets cannot contain additional information that is not already contained in the two-dimensional data sets; nevertheless, the more detailed and clearer these three-dimensional representations are, the easier it is for an attending physician to quickly recognize the extent of a pathological change. This is especially significant in the field of cranio-maxillofacial surgery in light of the complex anatomical interrelationships prevailing in the skull. Thus, here in particular, three-dimensional representations can help the attending surgeon to promptly spot bone fractures and other possible anomalies in the bones of the face and to plan surgical procedures accordingly.

This becomes clear by considering the images of Patient 1 , who suffered a complex centro-lateral mid-face fracture (Figures 1-4). Three-dimensional depictions enable the attending physician to quickly get an overview of the injured structures, since this entails viewing only a single image instead of scrolling through many different layers in various levels of two-dimensional cross-sectional images. In this case, the three-dimensional reconstruction also makes it possible to immediately begin considering how to proceed operatively, which osteosynthesis material is to be used, and where it should be placed.

Three-dimensional representations can also be very useful at morning briefings in conjunction with shift changes by providing a clear indication of the extent of a patient's injuries in the form of a few three-dimensional images. When, in the future, it becomes possible to generate three-dimensional reconstructions in real time automatically, this could be a useful tool to provide the team delivering interdisciplinary shock-room treatment with a quick, plastic overview of the injured structures in the case of a complex facial trauma. A comparison of the images of this patient generated by volume rendering (Figures 1 and 2) and Cinematic Rendering (Figures 3 and 4) demonstrates that the latter appear more lifelike and that, due to their better ambient occlusion and clearer depiction, they make it easier to determine which bony structures are broken. It is also simpler to visually differentiate the individual fragments from each other, and to assess whether the fractured bone components have been rotated or tilted, and, if so, in which direction.

Until now, however, it has been difficult to substantiate that Cinematic Rendering is superior to Volume Rendering in facilitating clinical diagnoses of bone injuries in everyday practice in the field of cranio-maxillofacial surgery. Dappa, Higashigaito [7] et al. found no major differences with respect to arriving at clinical diagnoses, and, as far as we know, there have been no scientific studies on the subject of the added diagnostic value of Cinematic Rendering in comparison to Volume Rendering.

Nevertheless, in the case of Patient 2, who suffered fractures of the mandible in the area of the right angle of the jaw as well as in the area of Tooth 33, we found that there was indeed a significant improvement in the process of arriving at a diagnosis with the help of Cinematic Rendering. On the reconstructions of the mandible generated by volume rendering (Figures 5 and 6), the fracture in the area of the right angle of the jaw is indeed clearly visible, whereas the fracture in the area of Tooth 33 does not come to light. They reveal neither interruptions of continuity nor other irregularities in the area of the cortical bone in this region, so that no signs of a fracture are visible. In comparison to them, the three-dimensional representations generated with Cinematic Rendering of the same patient's data (Figures 7 and 8) are not only more lifelike and realistic; both fractures are also clearly visible on them. One can recognize an obvious interruption of continuity of the mandible in the area of Tooth 33 (see arrow) indicating the mobile fracture near it. The fact that this fracture is visible here is possibly attributable to the greater detail and sharpness of the image, to the more complex illumination, and to the better ambient occlusion of Cinematic Rendering. This ambient occlusion makes indentations and clefts appear darker since ambient light is less able to penetrate them.

Due to their sharper images and improved ambient occlusion, the three-dimensional reconstructions generated via Cinematic Rendering are also better suited for planning surgical procedures. For example, in the three-dimensional reconstructions of Patient 3, who suffered a fracture of both mandibular condyles (Figures 9-14), the reconstructions generated with Cinematic Rendering are especially well suited to assessing the bone fractures, since the images' sharpness enables the surgeon to determine whether the fractured bone portions can successfully be operatively joined. Moreover, the extent of the tilting of the fractured ends can also be determined. On the basis of these parameters, the surgeon can better plan the operative procedure and weigh such considerations as which type of osteosynthesis material (e.g. mini-osteosynthesis plate, lag screw osteosynthesis) should be used and which operative access path (e.g. intraoral, extraoral) is most appropriate.

With Cinematic Rendering, it is also possible to use various transfer functions, depending upon which type of tissue is to be visualized (e.g. 
soft tissue, bone, blood vessels filled with contrast medium) [5]. In our work, we opted for functions that are particularly well suited to visualizing bone structures.

As with Volume Rendering, Cinematic Rendering also makes it possible to freely move three-dimensionally about the object or to "fly through" a three-dimensional reconstruction. In doing so, the observer can focus on the structures that are of particular interest. This can be seen especially well in Figures 10 and 12 in which the focus is on the right mandibular joint. Nevertheless, due to the high-definition graphics and the tremendous computer processing power required to achieve them, rendering in real time and changing the point of view in real time is dependent on the used hardware. Normally, it needs several seconds until the image that emerges is fully rendered and depicted in the highest possible quality [5].

Furthermore, Cinematic Rendering is a topic for scientific investigation, as well as education [15].

More clinical studies investigating the actual diagnostic advantages of Cinematic Rendering are certainly needed. But if we consider the photorealistic, detailed three-dimensional reconstructions that can be generated with Cinematic Rendering and the benefits provided by them, then it seems that Cinematic Rendering will be a promising post-processing method in the field of cranio-maxillofacial surgery on traumatic injuries [16,17].

\section{Conclusion}

Cinematic Rendering is a recently introduced post-processing method to generate three-dimensional reconstructions from twodimensional data sets produced by procedures such as computed tomography and magnetic resonance imaging. Cinematic Rendering significantly increases the quality of the three-dimensional postprocessing; in contrast to standard Volume Rendering, it is possible to produce nearly photorealistic, detailed three-dimensional representations of the human body. Thus, especially for craniomaxillofacial surgeons treating traumatic injuries, Cinematic Rendering seems to make it easier to diagnose fractures and abnormalities and to plan the corresponding operative procedures.

\section{Disclosure}

No conflicts of interest. No grants or financial supports.

\section{References}

1. Calhoun PS, Kuszyk BS, Heath DG, Carley JC, Fishman EK (1999) Three-dimensiona volume rendering of spiral CT data: theory and method. Radiographics 19: 745-764.

2. Drebin RA, Carpenter L, Hanrahan P (1988) Volume rendering. Comput Graph 22: 65-74.

3. Fishman EK, Drebin B, Magid D, Scott WW Jr, Ney DR, et al. (1987) Volumetric rendering techniques: applications for three-dimensional imaging of the hip. Radiology 163: 737-738

4. Levoy M (1988) Display of surfaces from volume data. IEEE Comput Graph Applicat 8: $29-37$

5. Fellner FA (2016) Introducing cinematic rendering: A novel technique for postprocessing medical imaging data. J Biomedical Science and Engineering 9: 170-175.

6. Fishman EK, Ney DR, Heath DG, Corl FM, Horton KM, Johnson PT (2006) Volume rendering versus maximum intensity projection in CT angiography: what works best, when, and why. Radiographics 26: 905-922.

7. Dappa E, Higashigaito K, Fornaro J, Leschka S, Wildermuth S, Alkadhi H (2016) Cinematic rendering - an alternative to volume rendering for 3D computed tomography imaging. Insights Imaging 7: 849-856.

8. Luccichenti G, Cademartiri F, Pezzella FR, Runza G, Belgrano M, et al. (2005) 3D reconstruction techniques made easy: know-how and pictures. Eur Radiol 15: 21462156.

9. Ebert LC, Schweitzer W, Gascho D, Ruder TD, Flach PM, Thali MJ, Ampanozi G (2017) Forensic 3D Visualization of CT Data Using Cinematic Volume Rendering: A Preliminary Study. AJR Am J Roentgenol 208: 233-240.

10. Comaniciu D, Engel K, Georgescu B, Mansi T (2016) Shaping the future through innovations: From medical imaging to precision medicine. Med Image Anal 33: 19-26.

11. Kroes T, Post FH, Botha CP (2012) Exposure render: an interactive photo-realistic volume rendering framework. PLoS One 7: e38586.

12. Debevec PE (1998) Rendering Synthetic Objects into Real Scenes: Bridging Traditinal and Image-Based GRaphics with Global Illumination and High Dynamic Range Photography. SIGGRAPH 98: 189-198.

13. Mamassian P, Knill DC, Kersten D (1998) The perception of cast shadows. Trends Cogn Sci 2: 288-295.

14. Langer MS, Bulthoff HH (2000) Depth discrimination from shading under diffuse lighting. Perception 29: 649-660.

15. Fellner FA, Engel K, Kremer C (2017) Virtual Anatomy: The Dissecting Theatre of the Future-Implementation of Cinematic Rendering in a Large $8 \mathrm{~K}$ High-Resolution Projection Environment. Journal of Biomedical Science and Engineering 10: 367-375.

16. Rowe SP, Zinreich SJ, Fishman EK (2018) New and emerging patient-centered CT imaging and image-guided treatment paradigms for maxillofacial trauma. Emerg Radiol 25: 533-545.

17. Stadlinger B, Valdec S, Wacht L, Essig H, Winklhofer S (2020) 3D-cinematic rendering for dental and maxillofacial imaging. Dentomaxillofac Radiol 49: 20190249.

Copyright: (C2020 Stehrer R. This is an open-access article distributed under the terms of the Creative Commons Attribution License, which permits unrestricted use, distribution, and reproduction in any medium, provided the original author and source are credited. 